\title{
Type II muscle fibers atrophy associated with silent corticotroph adenoma in a dog
}

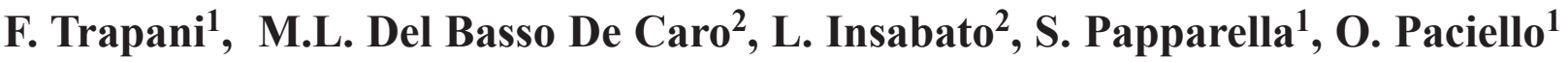 \\ ${ }^{1}$ Department of Pathology and Animal Health, Laboratory of Comparative Neuromuscular Diseases; \\ University of Naples Federico II, via Delpino, 180137 Naples - Italy \\ ${ }^{2}$ Biomorphological and Functional Science, Section of Anatomic Pathology University of Naples \\ Federico II, Naples-Italy.
}

\begin{abstract}
The Silent Corticotroph Adenoma (SCA) is a pituitary adenoma variant characterized by the immunoreactivity for adrenocorticotropic hormone (ACTH) and related peptides, without the clinical signs of Cushing's disease. SCA has been postulated to either secrete structurally abnormal ACTH that is inactive but detectable by immunohistochemistry or radioimmunoassay, or to secrete ACTH intermittently or at low levels continuously. Excess of ACTH has been associated to type II muscle atrophy. We describe a case of type II muscle fibers atrophy associated with silent corticotroph adenoma in a dog. The dog showed moderate to severe proximal muscle wasting and weakness with normal levels of muscle-associated enzymes. In the limb muscle biopsies, type II fibers were uniformly smaller than type I fibers. In temporalis muscles, there were few atrophic fibers, and several irregular areas of loss of enzymatic activity observed in NADH, SDH and COX stains. The tumour showed a trabecular growth pattern and immunohistochemical analysis demonstrated the presence of cytoplasmic immunoreactivity for ACTH. The muscle atrophy was considered to be related to an excess of inactive ACTH. Studying spontaneous occurring rare diseases in animals could help to understand the mechanism of similar diseases in human has well.
\end{abstract}

Keywords: type II muscle fiber atrophy, silent corticotroph adenoma, ACTH, dog

\section{Introduction}

In humans, pituitary adenomas are classified into two large groups of tumours: clinically functioning and non-functioning adenomas, according to whether or not an endocrine syndrome is present [1]. A third of all pituitary adenomas are not associated with either clinical or biochemical evidence of hormone excess. The Silent Corticotroph Adenoma (SCA) is a pituitary adenoma variant characterized by the immunoreactivity for adrenocorticotropic hormone (ACTH) and related peptides such as $\beta$-endorphin an $\beta$-lipotrophin, despite the fact that the patients with such adenoma don't show clinical signs of Cushing's disease or increased serum levels reflecting excess ACTH secretion [1].

SCA is morphologically indistinguishable from functioning corticotroph adenoma, therefore a complete immunohistochemical analysis of the tumour associated

Correspondence: O. Paciello, Dept. of Pathology and Animal Health, Laboratory of Comparative Neuromuscular Diseases, University of Naples Federico II, Via Delpino,1 80137, Naples, Italy; tel.: (+39) 0812536466, fax.: (+39) 0812536186, e-mail: paciello@unina.it with clinical evaluation is essential for an accurate diagnosis [1]. SCA has been postulated to either secrete structurally abnormal ACTH that is inactive but detectable by immunohistochemistry or radioimmunoassay, or to secrete ACTH intermittently or at low levels continuously [2]. In veterinary medicine SCA is rarely described probably because of the lack of specific clinical signs and biochemical characterization.

Dogs with SCA are depressed, show incoordination, weakness and progressive loss of weight and muscle atrophy [3].

Proximal weakness, especially of the pelvic limbs, is a well recognized complication of endocrine disorders [4]. The most consistent change both during Cushing's syndrome as well as in steroid myopathy is a selective type II muscle fibers atrophy [4] but, to our knowledge, a case of SCA associated with type II muscle fibers atrophy has never been described.

\section{Materials and methods}

The dog was a pit bull, male, 5 years old, showed moderate to severe proximal muscle wasting and weakness, difficulty in stepping stairs and slight myalgia. The serum levels of muscle-associ- 
ated enzymes Creatine kinase (CK), Lactate dehydrogenase (LDH), Aspartate Aminotransferase (AST) were in the normal range. No specific constitutional signs of Cushing's syndrome were present and serum cortisol level was normal. Further, the ACTH stimulation test and the low dose dexamethasone suppression test didn't show any particular alteration of the adrenal gland. A total body radiological exam and echocardiography were performed but no significant alterations were seen. By contrary, at the necropsy a $4 \mathrm{~cm}$ in diameter tumour mass was observed in the sella turcica, under the base of the brain (Fig. 1). On cut surface it showed a brownish appearance and focal areas of haemorrhages. The brain including the tumour was promptly fixed in $10 \%$ buffered formalin. Sections were stained with haematoxylin and eosin to establish histological diagnosis. For the immunohistochemistry, the peroxidase-antiperoxidase technique was used. Paraffin sections 4-6 $\mu \mathrm{m}$ in thickness were immunostained for $\mathrm{GH}$, PRL, LH, FSH, TSH, $\beta$-endorphin and ACTH (Polyclonal antibodies prediluted, Cell Marque Rocklin, CA, USA, and for Ki67/MIB1 to evaluate the tumour growth characteristics (diluted 1:100, DakoCytomation, Glostrup, Denmark). Negative controls were performed by incubating the sections with the same dilution of normal rabbit serum as those used for the corresponding specific antiserum. Samples from quadriceps and temporalis muscles were taken and frozen in isopentane precooled in liquid nitrogen and stored at $-80^{\circ} \mathrm{C}$. Sections from these specimens were stained using histological and histochemical techniques routinely used in our laboratory and well characterized in veterinary myology [5], in particular: haematoxylin

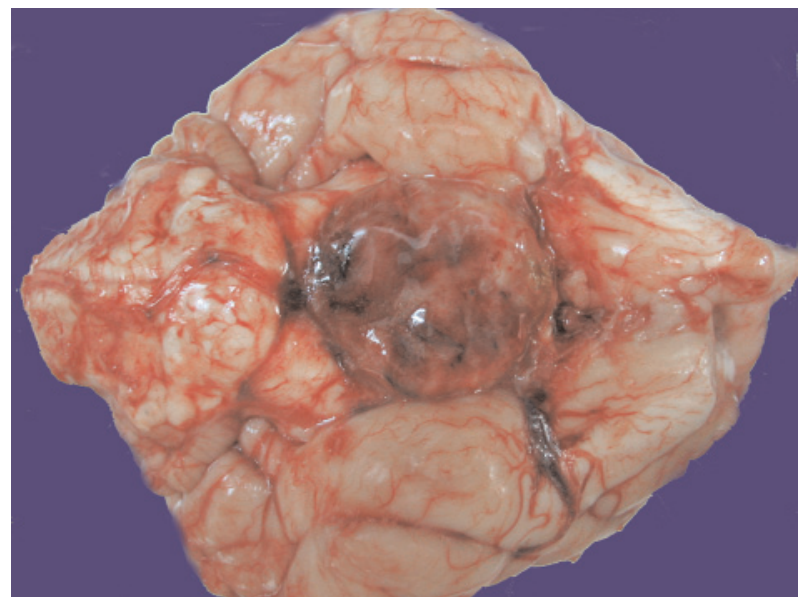

Fig. 1. Brain, dog. A large tumour is present in the sella turcica, under the base of the brain. It shows brownish appearance and focal areas of haemorrhages.

and eosin (HE), Engel trichrome, reduced nicotinamide-adenine dinucleotide tetrazolium reductase (NADH-TR), succinate dehydrogenase $(\mathrm{SDH})$, cytochrome oxidase $(\mathrm{COX})$, and the myofibrillar Adenosine Triphosphatase (ATPase) reactions at $\mathrm{pH} 9.4$ and 4.3 .
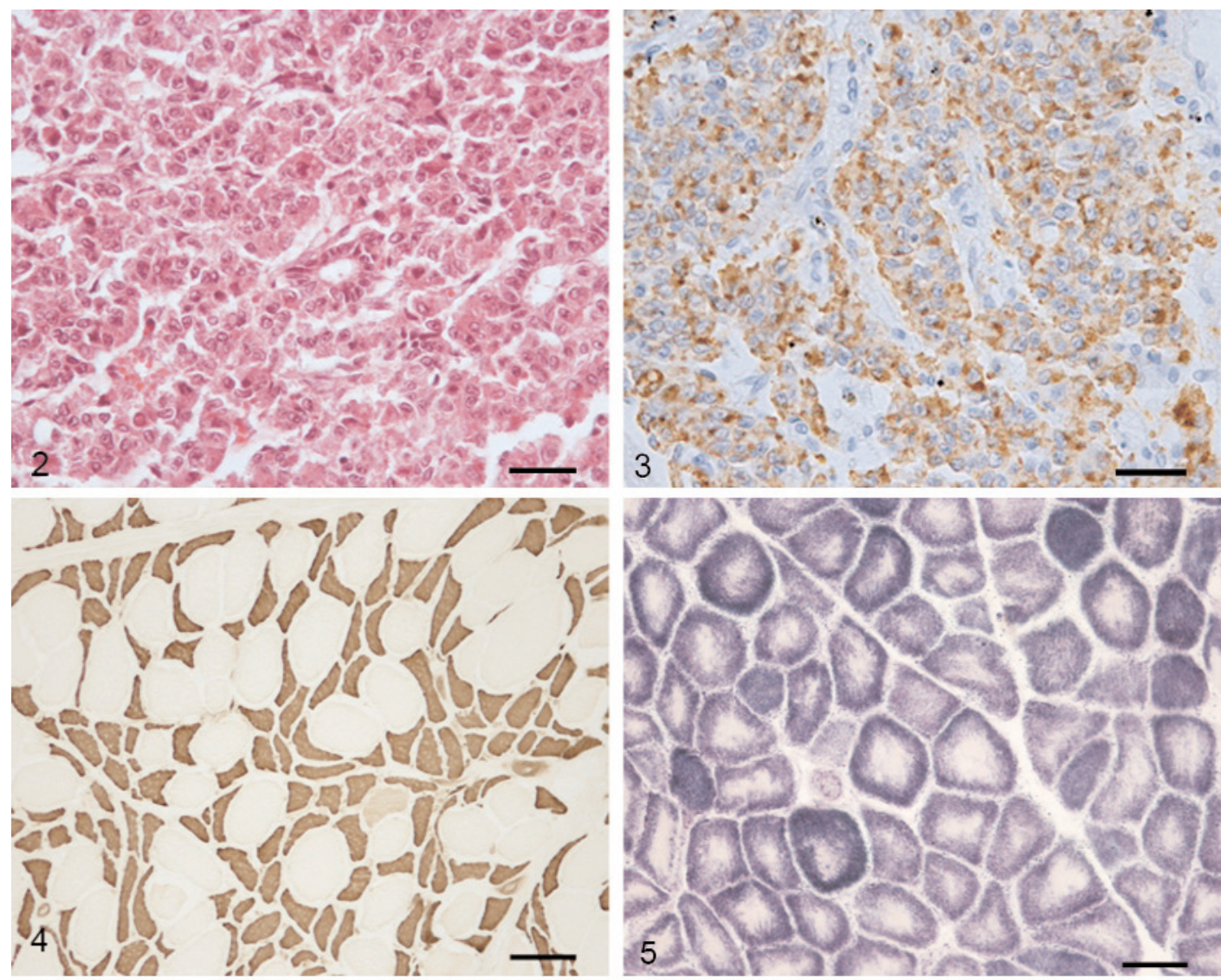

Fig. 2. Brain, macroadenoma, dog. The tumour shows trabecular morphologic growth pattern with cells that have very distinct cytoplasm borders and tend to touch each other in a "tile-like" arrangement. HE stain. Bar $=44 \mu \mathrm{m}$. Fig. 3. Brain, macroadenoma, dog. Cytoplasmic immunoreactive granules for ACTH. Immunohistochemistry, peroxidase-antiperoxidase technique. Bar $=44 \mu \mathrm{m}$. Fig. 4. Skeletal muscle from limb; dog. The type II fibers (dark) are uniformly smaller that type I (light). ATPasi PH 9,4 stain. Bar $=20 \mu \mathrm{m}$. Fig. 5. Skeletal muscle from temporalis; dog. The majority of the fibers show irregular areas of loss of enzymatic activity. NADH-DR stain. Bar $=20 \mu \mathrm{m}$. 


\section{Results}

By light microscopy, the tumour showed a trabecular growth pattern (Fig. 2). The tumour cells had sharp cytoplasmic borders, in a "tile-like" fashion. The cytoplasms were granular and eosinophilic (Fig. 2). The nuclei were large with coarse chromatin.

Immunohistochemical analysis demonstrated the presence of cytoplasmic immunoreactivity for ACTH (Fig. 3) and scattered cells positive to $\beta$-endorphin (data not showed). The tumour was negative for all other antibodies tested. For Ki-67 evaluation, 5 highpower fields $(\times 40)$ with the highest density of positive nuclei were examined and a mean of 500 tumour cells was counted. The result was expressed as percentage of tumour cells with positive nuclei. Only nuclei with a strongly positive label were counted. The tumour showed increased Ki67/MIB1 labelling index exceeding $3 \%$. Basing on the histological pattern and the clinical data a diagnosis of silent corticotroph adenoma was made.

Histological studies of the muscles showed two different patterns of lesions. In the limb muscle biopsies, type II fibers, identify with the ATPase reactions at $\mathrm{pH}$ 4.3 and $\mathrm{pH} 9.4$, were uniformly smaller than type I fibers and were at varying stages of atrophy (Fig. 4), some Ragged Red fibers were seen on Engel trichrome staining; NADH-TR, COX and SDH showed many type I fibers with a intensely stained mitochondria. In temporalis muscles, on the ATPase stains, only few type II atrophic fibers were seen, and several irregular areas of loss of enzymatic activity were observed in NADH SDH and COX stains (Fig. 5).

\section{Discussion}

A changing pattern in hormonal expression and secretion by pituitary adenomas is a well recognized phenomenon and the associated muscle II fibers atrophy consistent with that described in other species [6]. In humans and cats, atrophy is only observed in type IIB myofibers. Dogs, however, do not have classical type IIB myofibers and atrophy affects type IIA oxidativeglycolytic fibers [7]. A similar pattern of muscle fiber atrophy is found in steroid-induced myopathy in other species [6].

The primary mechanisms of how either excessive endogenous or exogenous corticosteroids can result in muscle weakness and atrophy appear to be through impairment of muscle protein balance resulting from decreased synthesis of soluble and myofibrillar proteins $[4,6]$. Corticosteroids are also known to depress glycolysis and induce an insulin resistant state. The selective vulnerability of type II fibers to atrophy may be explained by the fact that type II myofibers are more dependant on glycolysis than are type I myofibers $[4,6]$.
Furthermore, in temporalis muscles, core-like areas were observed in the oxidative enzyme stains (NADHTR, SDH, COX). These findings may reflect mild loss of myofibrils and altered distribution of mitochondria. The different morphological response of temporalis muscle may reflect the difference in composition from limb muscles such as they be selectively involved in masticatory muscle myositis [7-10].

In our case, because of the normal adrenal function detected by hormones assay and functional tests, ACTH does not induce a cortisol excess and may be, by itself, involved in the type II muscle fibre atrophy [4].

Several biologic mechanisms have been proposed to account for the discordance between the finding of ACTH immunoreactivity in tumour cells and the apparent lack of a corresponding Cushing's syndrome. These include the production of functionally inactive proopiomelanocortin derivates, which is either due to faulty translation or inadequate posttranslation processing [11]. Alternatively, secretion of ACTH by these tumours may be intermittent and/or serum ACTH levels so subtly elevated as to escape recording. It should be related to the failure exocytosis and increased intracellular disposal, by lysosomes or a defective packing into secretory granules due to inadequately developed Golgi apparatus [12].

For these reasons, despite type II fibers atrophy is generally attributed to glucocorticoid excess, an ACTH excess has been hypothesized to have myophatic actions that are separate from those of glucocorticoids but such pathogenic mechanism of ACTH remains obscure [4].

In conclusion, this case suggest that, in absence of specific clinical signs and biochemical changes, the presence of muscle II fibers atrophy can be related to an excess of inactive ACTH production occurring during SCA, if so, this results could help to understand similar disorders in human medicine as well.

\section{References}

[1] Beatriz M. and Lopes S., Tumors of the pituitary gland. In: Diagnostic histopathology of tumors. Ed. C.D.M. Fletcher. 2nd Ed.; Curchill Livingstone, UK 2000, Vol. II pp. 933-945.

[2] Lopez JA, Kleinschmidt-Demasters Bk B, Sze CI, Woodmansee WW, Lillehei KO. Silent corticotroph adenomas: further clinical and pathological observations. Hum Pathol. 2004;35(9):1137-47.

[3] Capen CC. Endocrine glands. In : Pathology of domestic animals. $5^{\text {th }}$ Ed. Jubb, Kennedy and Palmer. Vol III, Elsevier, 2007; p. 343.

[ 4] Ubogu EE, Ruff RL, Kaminski HJ. Endocrine myopathies in: Myology. Ed. Engel AG and Franzini-Amstrong C. 3th ed., Vol II 2004; pp. 1713-1738. McGraw-Hill, USA.

[5] Paciello O, Papparella S. Histochemical and immunohistological approach to comparative neuromuscular diseases. Folia Histochem Cytobiol. 2009;47(2):143-52.

[6] Aleman M, Watson J, Williams D, LeCouteur Nieto J, Shelton G. Myopathy in horses with pituitary pars intermedia dys- 
function (Cushing's disease) Neuromuscul Disord. 2006; 16,(11):737-744.

[7] Toniolo L, Maccatrozzo L, Patruno M, Pavan E, Caliaro F, Rossi R, Rinaldi C, Canepari M, Reggiani C, Mascarello F. Fiber types in canine muscles: myosin isoform expression and functional characterization. Am J Physiol Cell Physiol. 2007; 292(5):C1915-1926.

[ 8] Orvis JS, Cardiriet GH: Canine muscle fiber types arid susceptibility of masticatory muscles to myositis. Muscle and Nerve. 1981;4:354-359.

[ 9] Shelton GD, Bandrnan E, Cardinet GI: Electrophoretic comparison of myosins from masticatory muscles and selected limb muscles in the dog. Am J Vet Rcs. 1985;46:493-498.
[10] Paciello O, Shelton GD, Papparella S, Expression of major histocompatibility complex class I and class II antigens in canine masticatory muscle myositis. Neuromuscul Disord. 2007;17(8):662.

[11] Sahli R, Christ ER, Seiler R, Kappeler A, Vajtai I. Clinicopathologic correlations of silent corticotroph adenomas of the pituitary: report of four cases and literature review. Pathol Res Pract. 2006;202(6):457-64.

[12] Daems T, Verhelst J, Michotte A, Abrams P, De Ridder D, Abs R. Modification of hormonal secretion in clinically silent pituitary adenomas. Pituitary. 2009;12(1):80-6.

Submitted: 14 March, 2010 Accepted after reviews: 27 April, 2010 